J. Clin. Chem. Clin. Biochem.

Vol. 15, 1977, pp. 691-697

\title{
Mathematische Analyse der Fehlerverteilungen bei Flow-Systemen und Konsequenzen für die Be- stimmung neuer methodenspezifischer Kontrollgrenzen
}

\author{
Von Astrid Domesle, K. Harm und W. Rehpenning \\ Abteilung für Medizinische Dokumentation und Statistik und Zentrallabor der Medizinischen Kliniken (Prof. Dr. \\ K. D. Voigt) des Universitäts-Krankenhauses Hamburg-Eppendorf
}

(Eingegangen am 20. Mai/22. Juli 1977)

\begin{abstract}
Zusammenfassung: Zur Bestimmung methodenspezifisch angepaßter Kontrollgrenzen für den Technicon Autoanalyzer SMA $12 / 60\left(\mathrm{Na}^{+}, \mathrm{K}^{+}, \mathrm{Cl}^{-}\right.$, Gesamt-Eiweiß, Albumin, P, Cholesterin, Harnstoff-N, Calcium, Kreatinin, Bilirubin, Harnsäure) wird versucht, die Fehlerverteilungen der Kontrollvariablen formelmäßig darzustellen. Hierbei erweist sich die Entwicklung der Verteilungsdichten nach Orthogonalfunktionen (Gram-Charlier-Reihe Typ A) wegen der Oszillationen an den Rändern der Verteilungen als nicht brauchbar. Wesentlich bessere Ergebnisse werden durch einen modifizierten Ansatz einer Gram-Charlier'schen Reihe vom Typ C erhalten, wobei aber die auslaufenden Flanken der Verteilungen, die für die Bestimmung der Fraktilen besonders wichtig sind, noch nicht optimal dargestellt werden können. Eine sehr befriedigende Approximation der empirischen Dichtefunktion wird erhalten, wenn die Kontrollvariablen als nichtadditive Uberlagerung zweier bzw. dreier normalverteilter Größen unterschiedlicher Streuung aufgefaßt werden. Hiermit können kanalspezifisch angepaßte Warn- und Kontrollgrenzen berechnet werden, die die bisher abgeprüften Parameter der konventionellen Qualitätskontrolle, die auf der Annahme von Normalverteilungen beruhen, ersetzen. Dadurch wird eine adäquate Überprüfung der Zuverlässigkeit von Flow-Systemen erreicht.
\end{abstract} Mathematical analysis of the error distributions in flow-systems and consequences for the determination of new
method-specific control limits

Summary: In connection with the determination of method adapted control limits for the Technicon Autoanalyzer SMA $12 / 60\left(\mathrm{Na}^{+}, \mathrm{K}^{+}, \mathrm{Cl}^{-}\right.$, Total Protein, Albumin, P, Cholesterol, Urea Nitrogen, Calcium, Creatinine, Bilirubin, Uric Acid) we have investigated the representation of the control variable error distributions by mathematical formulae. The application of orthogonal functions (Gram-Charlier's series type A) proved to be not practicable because of the oscillations occuring at the ends of the distributions. Considerably improved results were obtained by a modified expression of a Gram-Charlier's series of type C, although the tails of the distributions (which are particularly important for the calculation of the fractiles) could not be optimally approximated. However, a very satisfactory approximation of the empirical density functions was obtained when we interpreted the control variables as non-additive superposition of two or three normally distributed quantities with different variances. This enables us to calculate channel specific alarm and control limits, thereby replacing the conventional quality control parameters previously checked and based on the assumption of normal distributions. Thus, an adequate monitoring of the reliability of flow-systems can be achieved.

\section{Einleitung}

In unseren ersten statistischen Unter̦suchungen über die Verteilungen der Kontrolldaten $(1,2,3)$, die bei der ontime Kontrolle des SMA 12/60 mit unserem Laborinformationssystem „ELIAS“ $(4,5)$ anfielen, konnten wir bereits nachweisen, daß die Verteilungen der Kontrollvariablen erheblich von einer Normalverteilung abweichen. Dies zeigt sich insbesondere in einem großen positiven Exzeß in Verbindung mit lang auslaufenden
Flanken, wobei die Verteilungen jedoch im großen und ganzen als symmetrisch angesehen werden können. Damit wurden auch Ergebnisse von Lubran \& Martens (6) im Zusammenhang mit der Untersuchung hämatologischer Parameter bestätigt. Büttner (7) wies 1968 darauf hin, daß nur eine genaue Kenntnis der Fehlerstruktur die Grundlage für ein funktionierendes Kontrollsystem bieten kann und daß verschiedene statistische Tests, wie sie in der Qualitätskontrolle benutzt werden, nur bei Vorliegen von Normalverteilungen zulässig sind. Weil 
sich hieraus praktische Konsequenzen für die routinemäßige Uberwachung der Zuverlässigkeit von Patientendaten ergeben, ist es notwendig, die Verteilungstypen der Kontrollvariablen zu kennen. Sowohl die Berechnung kanalspezifisch „maßgeschneiderter" Kontrollgrenzen als auch die Entwicklung von Algorithmen, die den Vergleich von Präzision und Richtigkeit verschiedener Analysenserien gestatten, setzen die Analyșe der Fehlerverteilungen der Kontrollvariablen voraus. Diese soll im folgenden für die zwölf Methoden eines Technicon-Autoanalyzers SMA $12 / 60\left(\mathrm{Na}^{+}, \mathrm{K}^{+}, \mathrm{Cl}^{-}\right.$, Gesamt-Eiweiß, Albumin, P, Cholesterin, Harnstoff-N, Calcium, Kreatinin, Bilirubin, Harnsäure) ausführlich beschrieben werden.

\section{Material und Methoden}

Bei der Analyse der Fehlerverteilungen der Kontrollvariablen benutzten wir die in den letzten eineinhalb Jahren auf Datenträgern gespeicherten 65000 Einzeldaten der zur Driftkontrolle dienenden Referenzseren der Firmen Technicon und Hyland auf den Positionen 11, 21, 31 usw. (Tab. 1), die etwa die Hälfte

Tab. 1. Kontrollmuster für den Autoanalyzer SMA 12/60.

\begin{tabular}{cll}
\hline Position & Probe & Funktion \\
\hline $1-3$ & Referenzserum & Adaption Kalibration Standardlinie \\
$4-5$ & Monitrol I & Kontrolle Richtigkeit \\
6 & Monitrol I $\cdot$ X & Präzision von Tag zu Tag \\
7 & Monitrol II $\cdot$ X & Linearität \\
$11,21,31$ & Referenzserum & Driftkontrolle Standardlinie \\
$12,22,32$ & Referenzserum & Kalibration Standardlinie \\
$5 \times$ & Wasser & Kalibration Basislinie \\
$41-42$ & Referenzserum & Kalibration Standardlinie \\
$49-50$ & Monitrol I & Kontrolle Richtigkeit Kalibration \\
$51,61,71$ & Referenzserum & Driftkontrolle Standardlinie \\
$52,62,72$ & Referenzserum & Kalibration Standardlinie \\
\hline
\end{tabular}

aller Kontrollproben ausmachen. Um eine Aussage über das Ausmaß der kanalspezifischen Driften zu erhalten, wurden diese Proben unmittelbar vor jeder neuen Kalibration in die Probenkette eingefügt, da an dieser Stelle die größten Abweichungen vom Sollwert zu erwarten sind. Außerdem erlaubt dieses Vorgehen die Beurteilung der Zuverlässigkeit eines jeden Segmentes zwischen zwei Funktionseingriffen, da diese Seren auch als „Hilfsproben" sowohl für die Präzisions- als auch für die Richtigkeitskontrolle verwendet werden können. Auch der hohe Anteil an Kalibrierseren (Position 12, 22, 32 usw.) ist notwendig, um die Korrektur der kanalspezifischen Driften von Reagenzienund Standardkalibrationslinien für alle Methoden innerhalb vorgegebener Toleranzen (8) zu gewährleisten.

Während des Untersuchungszeitraumes würden folgende vom Hersteller angebotenen Standardmethoden benutżt:

Natrium, Kalium (Flammeñphotometrie), Chlorid (Quecksilberthiocyanat-Methode), Gesamt-Eiweiß (Biuret-Reaktion), Albumin (Bromkresolgrïn-Methode), anorganischer Phosphor (Molybdänblau-Reaktion), Cholesterin (Liebermann-BurchardReaktion), Harnstoff-Stickstoff (Diacetylmonoxim-Reaktion), Calcium (Cresolphthalein-Methode), Kreatinin (Jaffé-Reaktion), Bilirubin (Azobilirubin-Reaktion nach Jendrassik \& Gróf) Hänsäure (Phosphorwolframsäure-Methode).

Für alle Methoden mit Ausnahme voñ Bilirubin und Harnsäure wuruden etwa 5500 Einzelwerte in die mathematische Analyse der Verteilungstypen der Kontrollvariablen einbezogen.

Für die Analyse der Fehlerverteilungen ist die Behandlung von Extremwertën (Ausreißer) von fundamentaler Bedeutung (9). Für die statistische Auswertung ist es notwendig, Werte zu eliminieren, die offensichtlich nicht zu dem betrachteten Kollektiv gehören. Diese „Ausreißer“ wurden nicht anhand vorgebener Grenzen ausgeschlossen, sondern durch einen iterativen Algorithmus bestimmt: Nach Festlegung eines bestimmten Faktors $f$ wurden jeweils nach Berechnung von Mittelwert und Standardabweichung alle Werte gestrichen, die sich um mehr als das $\mathrm{f}$-fache der Standardabweichung vom Mittelwert unterschieden. Dieses Vorgehen wurde so oft wiederholt, bis keine weiteren Ausireißer mehr auftraten. Bei unserer ersten statistischen Untersuchung (1) arbeiteten wir unter der vorläufigen Annahme einer Normālverteilung mit dem Faktor $f=3,3$. Eine nähere Betrachtung der Ausreißer ergab, daß̧ eịn Teil der gestrichenen Werte auf technische Fehler wie Verstopfung der analytischen Einheiten, sowie unsachgemäße Handhabung der gefriergetrockneten Kontrollproben zurückzuführen war. Gleichzeitig mußßten wir feststellen, daß ein großer Teil der Extremwerte nicht erklärbar war und daher mit großer Wahrscheinlichkeit zur betrachteten Fehlerverteilung gehörte. Wir haben deshalb probeweise mit den Faktoren $\mathrm{f}=4$ und $\mathrm{f}=5$ gearbeitet.

Die Meßwerte wurden on-line von einem Telefunken-Rechner TR 86 erfaßt, die mathematische Analyse erfolgte auf dem Großrechner TR 440 des Rechenzentrums der Universität Hamburg.

Zur formelmäßigen Beschreibung der Fehlerverteilungen der Kontrollvariablen wurden die in Abbildung 1 dargestellten Dichtefunktionen benutzt. Zeile 1 enthält die Formel für die standardisierte Normalverteilung. Der Ansatz in Z̃eile 2 entspricht einer Gram-Charlier'schen Reihe vom Typ A (10), von der die ersten Glieder genommen wurden. Die Ausdrücke $\Phi^{\mathrm{III}}$, $\Phi^{\text {IV }}$ usw. entsprechen den Ableitungen der Dichte der normierten Normalverteilung. Die Konstanten $C_{3}, C_{4}$ usw. leiten sich von den zentralen Momenten ab. Die Formel in Zeile 3 stellt einen modifizierten Ansatz einer Gram-Charlier'schen Reihe vom Typ $\bar{C}$ dar (11). Bei der Formel in Zeile 4 gingen wir von der Annahme aus, daß zwei verschiedene Fehlerursachen vor-

\begin{tabular}{|l|l|}
\hline NORMALVERTEILUNG & \\
\hline $\begin{array}{l}\text { GRAM - CHARLIIE - } \\
\text { REIHE TYP A }\end{array}$ & $f(t)=\frac{1}{\sqrt{2 \pi} \exp \left(-\frac{t^{2}}{2}\right)}$ \\
\hline $\begin{array}{l}\text { GRAM - CHARLIER- } \\
\text { REIHE TYP C MODIFIIIERT }\end{array}$ & $f(t)+\frac{C_{3}}{3 !} \Phi^{(1(1)}(t)+\frac{C_{4}}{4 !} \Phi^{(1)}(t)+\ldots$ \\
\hline $\begin{array}{l}\text { MISCHUNG ZWEIER } \\
\text { NORMALVERTEILUNGEN }\end{array}$ & $f(t)=A \exp \left(-B t^{2}-C t^{4}-D t^{6}\right)$ \\
\hline
\end{tabular}

Abb. 1. Verteilungsdichten der standardisierten Fehlervariablen. 
handen sind, die sich nichtadditiv überlagern, d. h. wir nahmen eine Mischverteilung von zwei normalverteilten Größen an.

Die Berechnung der Verteilungsdichten erfolgte mit Hilfe hierfür geschriebener FORTRAN-Programme. Während die Bestimmung der Konstanten für die ersten beiden Ansätze keinerlei Schwierigkeiten bereitet, ist für die Anpassung der beiden letzten Dichtefunktionen ein iteratives Vorgehen notwendig. Bezeichnen wir die Abszissen mit $t_{i}$, die Ordinaten mit $y_{i}$ und geeignet gewählte Gewichte mit $g_{j}$, wobei $i=1, \ldots \ldots, K$ und $K$ die Anzahl der Klassen bedeutet, so ist die Summe:

$$
S=\sum_{i=1}^{K} g_{i}\left(y_{i}-f\left(t_{i}\right)\right)^{2}
$$

zu minimieren. Weil nur Werte zugelassen werden, die im $\pm 5 \mathrm{~s}$ Bereich liegen, gilt die Nebenbedingung:

$$
F=\int_{-5}^{5} f(t) d t=1
$$

Für den letzten Ansatz (Mischung zweier Normalverteilungen) folgt hieraus 2. B., daß nicht gilt:

$$
A+B=1 \text { sondern } A+B>1 \text {. }
$$

Wir haben daher sowohl A als auch B als freie zu bestimmende Konstanten betrachtet. Bei der Durchführung der Rechnungen zeigte es sich, daß die herkömmlichen Anpassungsalgorithmen (Newton-Methode (12) oder modifiziertes Marquardt-Verfahren (13)) nicht zum Ziele führten, da immer wieder Uberschreitungen des Rechenbereiches (Exponentenüberlauf) auftraten. Als überlegen und effizient erwies sich dagegen die Simplex-Methode von Nelder \& Mead (14), die es zudem auf einfache Weise erlaubte, der Nebenbedingung Gl. 2 Rechnung zu tragen. Wir bestimmten zunächst Anfangswerte für die Iteration, indem wir den Algorithmus ohne die Nebenbedingung Gl. 2 anwandten. Danach setzten wir

$$
\mathrm{G}=\exp (65|\mathrm{~F}-1|)
$$

und minimierten $\mathbf{S}^{\prime}=\mathrm{G} \times \mathbf{S}$.

Es zeigte sich, daß die Flächenbedingung (GL 2) auf mindestens 6 geltende Ziffern erfüllt war. Die Gewichte $g_{i}$ berechneten wir nach der Formel:

$$
\mathrm{g}_{\mathrm{i}}=\frac{10}{0,05+\mathrm{y}_{\mathrm{i}}}
$$

um die Ränder der Verteilungen hervorzuheben:

Nachdem die Verteilungen dargestellt waren, konnte die Berechnung der in Tabelle 3 aufgefuhrten Fraktilen auf ein einfaches Nullstellenproblem zurückgeführt werden.

\section{Ergebnisse und Diskussion}

Grundbedingung für die mathematische Analyse der Fehlerverteilungen der Kontrollvariablen war

1. die Beibehaltung des in Tabelle 1 vorgestellten Probenzuordnungsmusters und

2. die zeitliche Stabilität der Verteilungen.

Daß Punkt 2 zufriedenstellend erfültt war, ergibt sich sowohl aus der Analyse der zeitlichen Verläufe der Kontrollvariablen als auch durch $\chi^{2}$-Teste. Abbildung 2 zeigt einen Ausschnitt aus dem zeitlichen Verlauf der Kontrollvariablen für Albumin. Die jeweils während eines Tages gemessenen Werte sind vertikal in einer Spalte gedruckt. Wenn mehrere Werte auf die gleiche Druckposition fielen, wurde dieses im Rechenprogramm abgefragt und durch die Ausgabe verschiedener Symbole kenntlich gemacht. Die auffallenden Stufen im Verlauf sind durch drei verschiedene Chargen bedingt. Die Pfeile in der Abbildung lokalisieren die beiden Chargenwechsel. Wie man sieht, ist der zeitliche Verlauf der Kontrolldaten innerhalb der einzelnen Chargen stabil.

Natürlich müssen für die Analyse der Fehler die Differenzen der Einzelwerte von den Sollwerten benutzt werden. Auch die graphische Darstellung dieser Differenzen zeigt über den ganzen Untersuchungszeitraum keine systematischen Unterschiede sowohl innerhalb, als auch zwischen den einzelnen Chargen der Referenzseren. Dieses gilt mit Ausnahme des Bilirubins (Abb. 3), dessen zeitlicher Verlauf innerhalb der ersten beiden Chargen so große Unregelmäßigkeiten aufwies, daß die zugehörigen Kontrolldaten gestrichen und nur ein Rest von 3314 Daten für die weitere statistische Auswertung benutzt werden konnte. Eine Erklärung für diese Unregelmäßigkeiten sind tagesspezifisch schwankende Differenzen zwischen Ist- und Sollwerten bei den sekundären Standardproben mehrerer Chargenuntermengen. Nachdem die Voraussetzungen für die mathematische Analyse

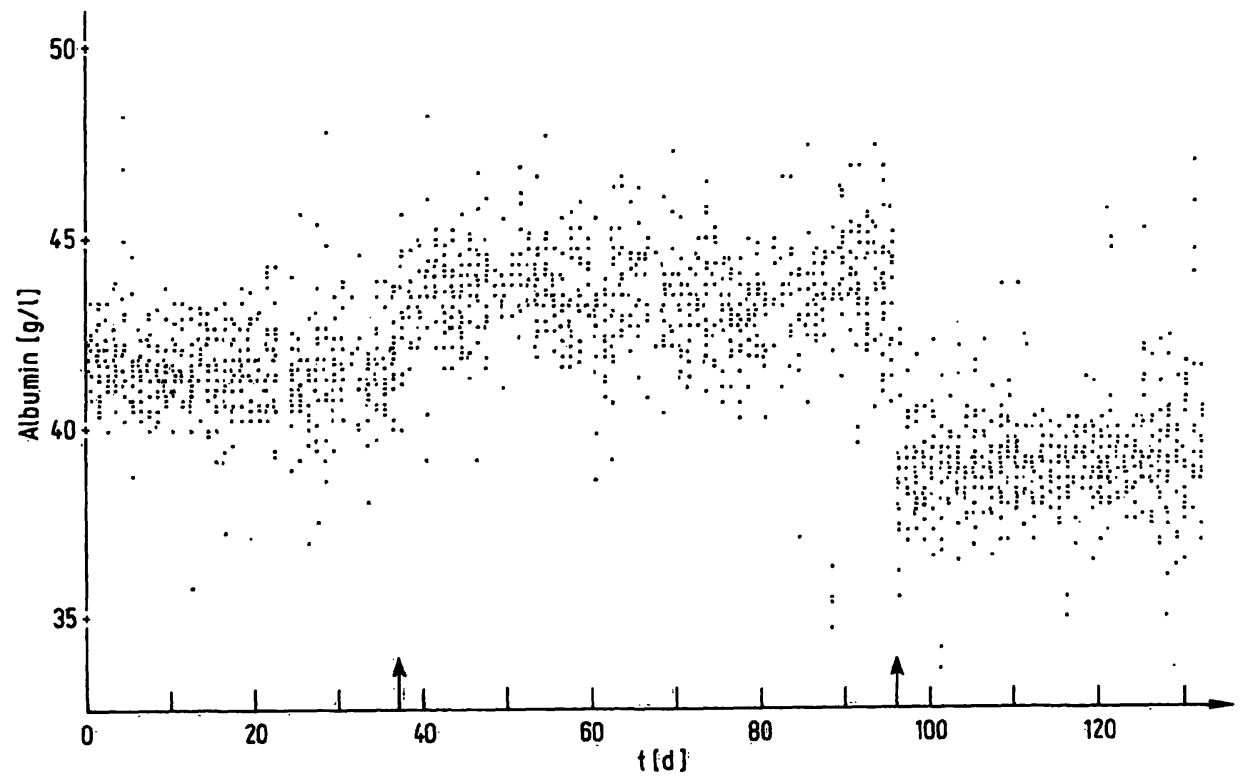

Abb. 2. Ausschnitt aus dem zeitlichen Verlauf der Kontrolldaten für Albumin. 


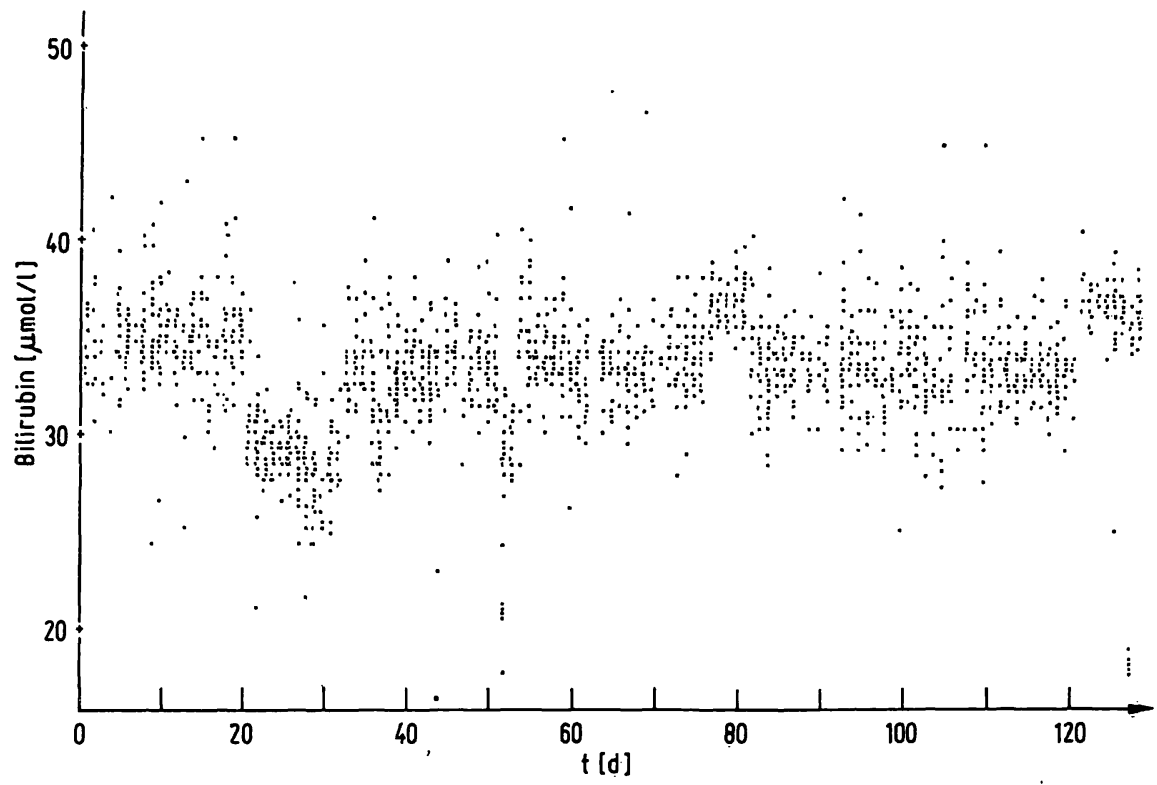

Abb. 3. Ausschnitt aus dem zeitlichen Verlauf der Kontrolldaten für Bilirubin.

der Kontrollvariablen erfüllt waren, versuchten wir als erstes, die Verteilungen der standardisierten und normierten Fehlervariablen durch die standardisierte Normalverteilung zu beschreiben. Hierzu wurden die Abweichungen von den Sollwerten in Form von Histogrammen dargestellt, in welche die zugehörigen Kurven der Normalverteilung projiziert wurden (Abb. 4). Die

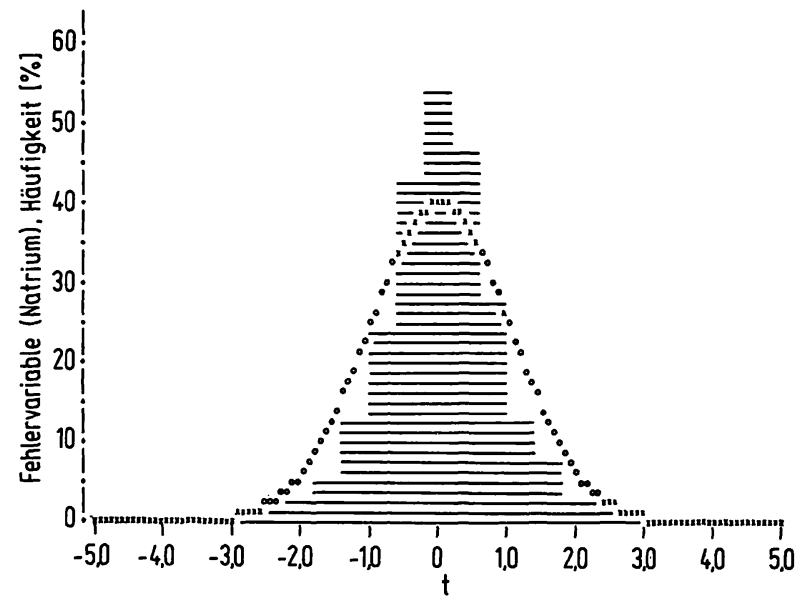

Abb. 4. Empirische Häufigkeitsverteilung der normierten und standardisierten Fehlervariablen $(=t)$ im Vergleich mit der entsprechenden Normalverteilung für Natrium.

Kreise in der Abbildung entsprechen einzelnen Punkten der theoretischen Kurve, Kreuze wurden gedruckt, wenn das Histogramm von der theoretischen Kurve durchsetzt war. Wie man am Beispiel des Natriums; das repräsentativ für alle Kanäle ist, sieht, wird die beobachtete Häufigkeitsverteilung nur sehr schlecht durch die Normalverteilung dargestellt.

Wir fanden, daß die Darstellung durch die ersten Glieder der Gram-Charlier'schen Reihe vom Typ A die Verteilungen durchgehend im Mittelteil besser beschreibt als die Normalverteilung. Die Approximation der Flanken hing dagegen stark davon ab, wie die Grenzen zum Ausschluß der Extremwerte gewählt wurden. So führte z. B. die Darstellung der Werte innerhalb des \pm 3,3 s-Bereiches zu einer befriedigenden Anpassung auch der Flanken. Schon unter Einbeziehung des \pm 4 s-Bereiches traten aber an den Rändern Oszillationen auf, die die Darstellung insgesamt unbrauchbar machten. Die Ergebnisse wurden noch unbefriedigender, wenn der $\pm 5 \mathrm{~s}$-Bereich mit einbezogen wurde (Abb. 5).

Bessere Anpassungen erzielten wir dann mit dem dritten Ansatz, der einer modifizierten Gram-Charlier'schen Reihe vom Typ $C$ entspricht. Die Approximation gelang für alle Werte innerhalb des $\pm 4 \mathrm{~s}$-Bereiches recht gut. Unter Einbeziehung des $\pm 5 \mathrm{~s}$-Bereiches zeigte eine detaillierte Untersuchung der Ränder der Verteilungen jedoch auch bei diesem Kurventyp noch systematische

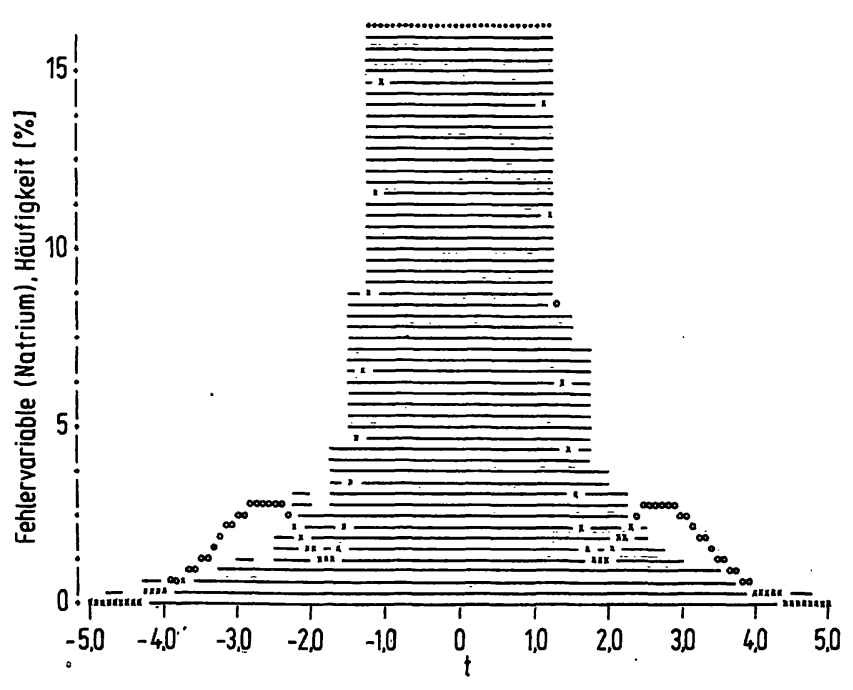

Abb. 5. Die Ränder der Verteilung nach der 2. Formel der Abbildung 1 (Gram-Charlier Typ A), vierfach vergrößert. 
Abweichungen vom theoretischen Verlauf (Abb. 6 und Abb. 7), so daß auch dieser Ansatz noch nicht optimal war. Ein zusätzliches, gravierendes Problem ergab sich dadurch, daß die berechneten Konstanten sehr stark davon abhängig waren, auf welche Weise wir den Wertebereich der Kontrollvariablen in Klassen unterteilt hatten. Einer der Autoren hatte diese Effekte an zweidimensionalen Problemen genauer untersucht und gefunden, daß Einflüsse dieser Art die Resultate beträchtlich verfälschen können (15). Diese zusätzliche Fehlerquelle konnten wir dadurch eliminieren, daß wir elf verschiedene Klasseneinteilungen vornahmen und die erhaltenen Histogramme ineinander mischten.

Eine wirklich befriedigende Darstellung, die auch die Ränder der Verteilungen exzellent approximierte, erhielten wir erst durch den vierten Ansatz, bei dem wir annahmen, daß die Fehler durch Mischung zweier, in ihrer Wirkung unabhängiger normalverteilter Größen unterschiedlicher Streuungen zustande kommen. In Abbildung 8 ist der Gesamtverlauf der so gewonnenen theoretischen Kurve und der durch Mischen der elf verschiedenen Histogramme entstandenen Werte der empirischen Dichte dargestellt. Einzelne Werte wurden

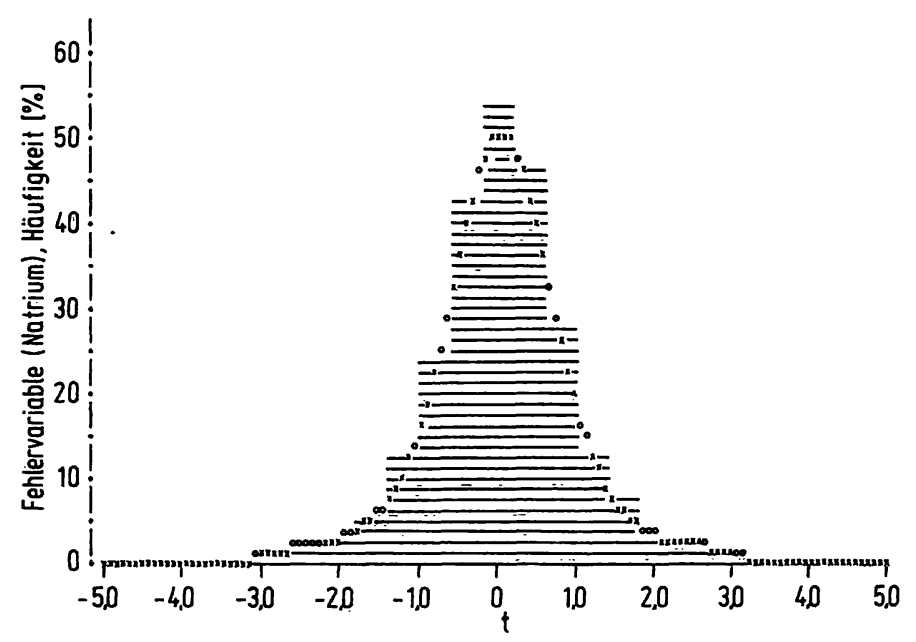

Abb. 6. Darstellung der empirischen Verteilungsdichte durch die 3. Formel der Abbildung 1 (Gram-Charlier Typ C modifiziert)

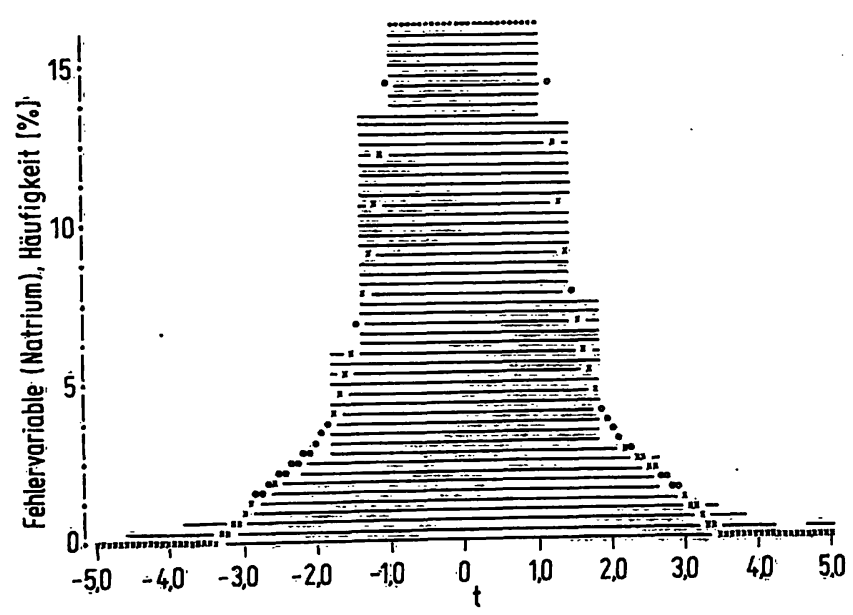

Abb. 7. Die Ränder der Ver̃teilung nach der 3. Formel der Abbildung 1 (Gram-Charlier Typ C modifiziert), vierfach vergrößert. durch Punkte wiedergegeben, die Uberlagerung mehrerer Punkte wurde durch unterschiedliche Symbole angezeigt. Die theoretische Kurve wurde durch Kreise gekennzeichnet, beim Zusammenfallen mit der vorliegenden Häufigkeitsverteilung wurden Sterne gedruckt. Abbildung 9 zeigt noch einmal die Dichte der Fehlerverteilung wie in Abbildung 8, jedoch mit der hineinprojizierten entsprechenden Normalverteilung. Daß auch die Ränder der

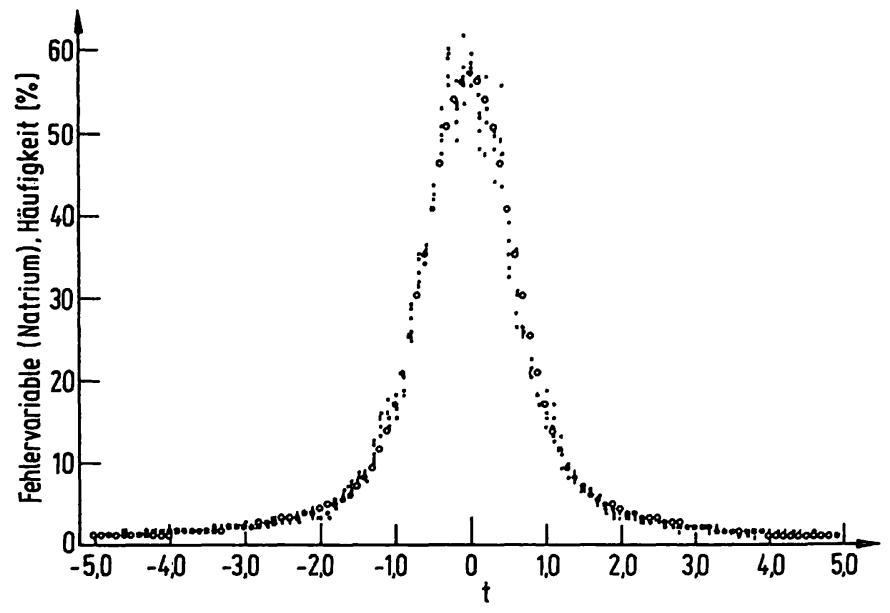

Abb. 8. Darstellung der empirischen Verteilungsdichte nach der 4. Formel der Abbildung 1. Einzelheiten siehe Text.

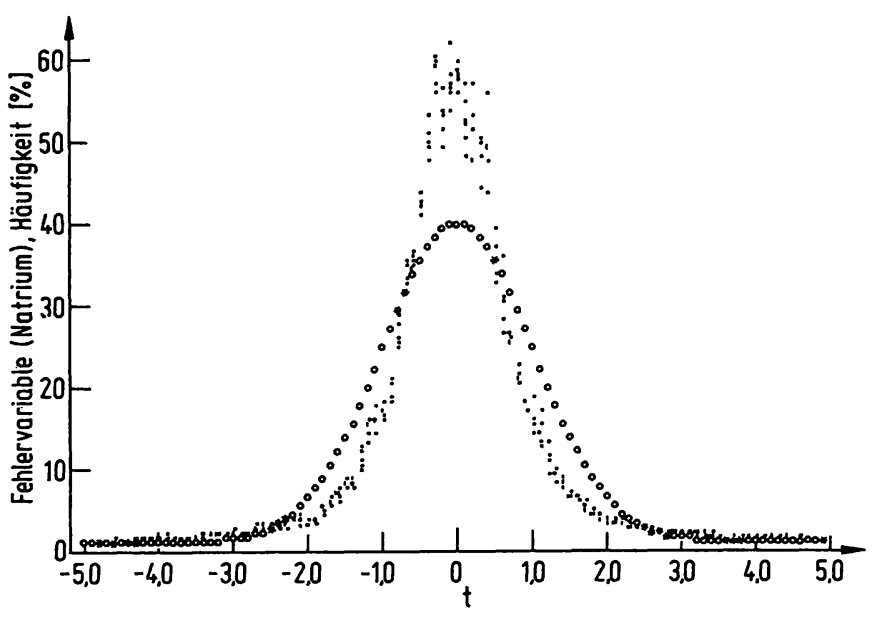

Abb. 9. Die Dichte der Fehlerverteilung wie in Abbildung 8 mit der hineinprojizierten entsprechenden Normalverteilung.

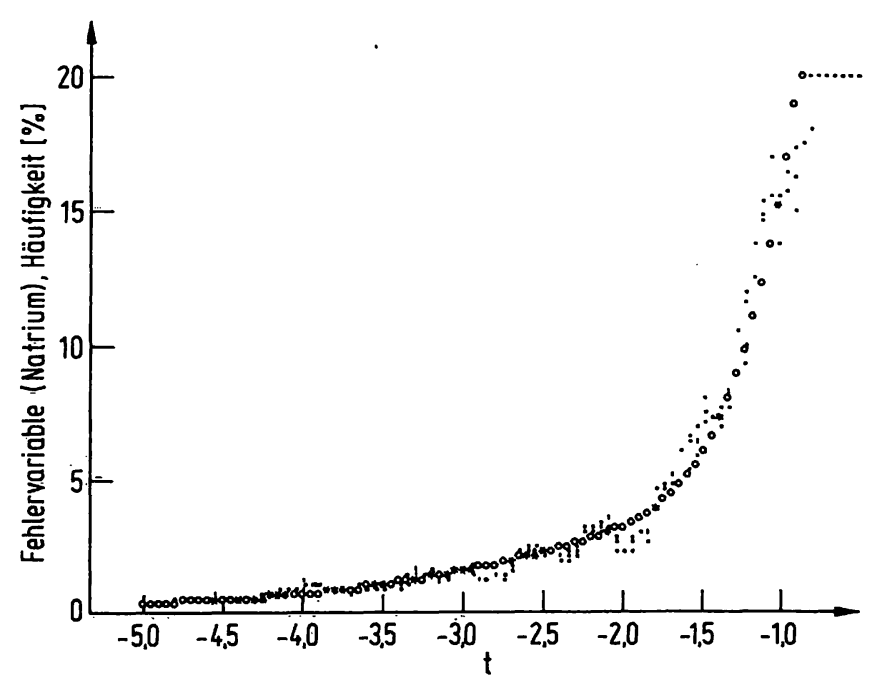

Abb. 10. Vergrößerter Ausschnitt der linken Flanke der in Abbildung $\mathbf{8}$ dargestellten Fehlerverteilung. 
Verteilungen hervorragend approximiert werden, erkennt man in Abbildung 10, die einen vergrößerten Ausschnitt zeigt.

Diese Form der Darstellung eignete sich für alle Kanäle bis auf Chlorid und Gesamt-Eiweiß, für die wir durch die Annahme einer zusätzlichen dritten Komponente die Darstellung noch signifikant verbessern konnten. Die Ergebnisse der Berechnungen sind in den Tabellen 2 und 3 zusammengefaßt. Die Spalten eins bis vier der Tabelle 2 enthalten Methode, Einheit, Anzahl der Meßwerte sowie die dazugehörigen Mittelwerte. Da die Bestimmung der Harnsäure erst zu einem späteren Zeitpunkt in das Methodenprogramm aufgenommen wurde, erklärt sich die im Vergleich zu den anderen Parametern wesentlich geringere Zahl an Kontrolldaten. Die Standardabweichung in Spalte fün wurde nach Abzug der Sollwerte berechnet. Die aus den gewonnenen Darstellungen berechneten Kontrollbereiche fur die Werte $p=0,95$, $\mathrm{p}=0,99$ und $\mathrm{p}=0,999$ sind in den Spalten sechs bis acht wiedergegeben. Die Faktoren für den $95 \%$, $99 \%$ und 99,9\%-Bereich, mit denen die Standardabweichung multipliziert werden muß, um auf die Kontrollbereiche zu kommen, sind in Tabelle 3 in den Spalten drei bis fünf dargestellt. Die Konstanten des letzten mathematiscien Ansatzes (Abb. 1) sind sukzessive in den Spalten sechs bis elf aufgefuhrt. Hierbei sind die Anteile der ver- schiedenen Normalverteilungen an der Gesamtverteilung in Prozent angegeben. Auf die Ursachen der Fehler kann an dieser Stelle nicht eingegangen werden. Immerhin kann aufgrund des vorliegenden Zahlenmaterials geschlossen werden, daß die Fehler nicht methodenabhängig, sondern systeminherent sind.

Betrachtet man die Faktoren für die 95\%-Grenzen, so erkennt man keine wesentlichen Unterschiede zum Faktor für die Normalverteilung, der 1,96 beträgt. Bei den 99\%-Grenzen, die im wesentlichen den Kontrollbereich determinieren, lassen sich bereits deutliche Abweichungen gegenüber dem bei der Normalverteilung vorliegenden Faktor von 2,58 feststellen.

Die Diskrepanz wird noch offensichtlicher, wenn man den Faktor von 3,29 für den 99,9\%-Bereich mit den neu berechneten Faktoren vergleicht.

Aus den bisherigen Ergebnissen ergeben sich folgende Konsequenzen für die Routineanalytik:

1. Bei der statistischen Qualitätskontrolle, die auf der Beurteilung von Einzelwerten beruht, kann bei Flow: Systemen nicht mehr von den auf der Normalverteilung beruhenden Kontrollgrenzen ausgegangen werden. Diese müssen vielmehr durch die neuen, kanalspezifisch angepaßten Warn- und Kontrollgienzen ersetzt werden.

Tab. 2. Statistische Kenngrößen und Vertrauensbereiche der Kontrollvariablen für die verschiedenen Parameter.

\begin{tabular}{|c|c|c|c|c|c|c|c|}
\hline Parameter & Einheit & $\mathbf{N}$ & Mittelwert & $\begin{array}{l}\text { Standard- } \\
\text { abweichung }\end{array}$ & $\begin{array}{l}\text { Bereich } \\
\mathrm{p}=0,95\end{array}$ & $\begin{array}{l}\text { Bereich } \\
p=0,99\end{array}$ & $\begin{array}{l}\text { Bereich } \\
p=0,999\end{array}$ \\
\hline Natrium & $\mathrm{mmol} / 1$ & 5525 & 145,3 & 1,422 & $142,3-148,3$ & $140,6-150,0$ & $139,0-151,6$ \\
\hline Kalium & $\mathrm{mmol} / \mathrm{l}$ & 5504 & 5,200 & 0,0660 & $5,067-5,333$ & $5,009-\quad 5,391$ & $4,943-\quad 5,455$ \\
\hline Chlorid & $\mathrm{mmol} / \mathrm{l}$ & 5518 & 104,6 & 1,682 & $101,2-108,0$ & $99,4-109,8$ & $97,5-111,7$ \\
\hline Gesamt-Eiweiß & $g / 1$ & 5430 & 65,17 & 1,912 & $61,04-69,30$ & $58,04-72,30$ & $56,03-74,31$ \\
\hline Albumin & $\mathrm{g} / 1$ & 5541 & 40,23 & 1,094 & $38,01-42,45$ & $37,07-43,39$ & $36,03-44,43$ \\
\hline Anorg. Phosphor $\mathrm{r}$ & $\mathrm{mmol} / \mathrm{l}$ & 5467 & 1,788 & 0,0341 & $1,720-\quad 1,856$ & $1,679-\quad 1,897$ & $1,639-\quad 1,937$ \\
\hline Cholesterin & $\mathrm{mmol} / \mathrm{l}$ & 5502 & 5,052 & 0,2918 & $4,460-5,644$ & $4,203-5,901$ & $3,920-6,184$ \\
\hline Harnstoff-N & $\mathrm{mmol} / 1$ & 5410 & 24,07 & 0,3464 & $23,35-24,79$ & $22,97-25,17$ & $22,58-25,56$ \\
\hline Calcium & $\mathrm{mmol} / 1$ & 5549 & 2,507 & 0,0592 & $2,388-2,626$ & $2,325-\quad 2,689$ & $2,258-2,756$ \\
\hline Kreatinin & $\mu \mathrm{mol} / 1$ & 5518 & 460,6 & 9,330 & $441,1-480,1$ & $430,7-490,5$ & $420,0-501,2$ \\
\hline Bilirubin & $\mu \mathrm{mol} / 1$ & 3314 & 43,76 & 1,896 & $39,95-47,57$ & $38,09-49,43$ & $36,12-51,40$ \\
\hline Harnsäure & $\mu \mathrm{mol} / 1$ & 1928 & 450,0 & 5,138 & $439,4-460,6$ & $433,4-466,6$ & $427,3 \quad-472,7$ \\
\hline
\end{tabular}

Tab. 3. Fraktilen und berechnete Konstanten der Kontrollvariablen.

\begin{tabular}{|c|c|c|c|c|c|c|c|c|c|c|}
\hline Parameter & Einheit & $f_{0,95}$ & $f_{0,99}$ & $f_{0,999}$ & $\mathrm{~A}[\%]$ & $\sigma_{1}$ & $\mathrm{~B}[\%]$ & $\sigma_{2}$ & $\mathrm{C}[\%]$ & $\sigma_{3}$ \\
\hline Natrium & $\mathrm{mmol} / \mathrm{l}$ & 2,13 & 3,28 & 4,41 & 70,75 & 0,5634 & 29,29 & 1,562 & - & - \\
\hline Kalium & $\mathrm{mmol} / \mathrm{l}$ & 2,01 & 2,89 & 3,87 & 56,22 & 0,5866 & 43,78 & 1,272 & - & - \\
\hline Chlorid & $\mathrm{mmol} / \mathrm{l}$ & 2,03 & 3,09 & 4,20 & 63,79 & 0,6066 & 30,84 & 1,451 & 5,38 & 0,1293 \\
\hline Gesamt-Eiweiß & $\mathrm{g} / \mathrm{l}$ & 2,16 & 3,73 & 4,78 & 59,70 & 0,7954 & 15,31 & 2,182 & 25,33 & 0,3387 \\
\hline Albumin & $g / 1$ & 2,03 & 2,89 & 3,84 & 53,53 & 0,5961 & 46,48 & 1,256 & - & - \\
\hline Anorg. Phosphor & $\mathrm{mmol} / \mathrm{l}$ & 1,99 & 3,19 & 4,37 & 77,12 & 0,6667 & 22,92 & 1,591 & - & - \\
\hline Cholesterin & $\mathrm{mmol} / \mathrm{l}$ & 2,03 & 2,91 & 3,88 & 54,70 & 0,5365 & 45,30 & 1,272 & - & - \\
\hline Harnstoff-N & $\mathrm{mmol} / \mathrm{l}$ & 2,08 & 3,18 & 4,31 & 70,65 & 0,6412 & 29,37 & 1,509 & - & - \\
\hline Calcium & $\mathrm{mmol} / \mathrm{l}$ & 2,01 & 3,07 & 4,20 & 71,69 & 0,6837 & 28,33 & 1,464 & - & - \\
\hline Kreatinin & $\mu \mathrm{mol} / 1$ & 2,09 & 3,21 & 4,35 & 72,54 & 0,6947 & 27,50 & 1,542 & - & - \\
\hline Bilirubin & $\mu \mathrm{mol} / 1$ & 2,01 & 2,99 & 4,03 & 64,05 & 0,5689 & 35,96 & 1,359 & - & - \\
\hline Harnsäure & $\mu \mathrm{mol} / 1$ & 2,07 & 3,24 & 4,42 & 76,94 & 0,7379 & 23,11 & 1,616 & - & - \\
\hline
\end{tabular}


2. Bei der Beurteilung einzelner Meßwerte wie z. B. von Patientendaten, muß von anderen Unsicherheitsfaktoren ausgegangen werden als dies bei der Annahme normalverteilter Fehler der Fall ist.

3. Die gezogenen Schlußfolgerungen gelten nicht mehr, wenn bei der Qualitätskontrolle mehrere Kontrolldaten zu Mittelwerten zusammengefaßt und zur Kontrolle benutzt werden. Dann ergibt sich nämlich nach dem zentralen Grenzwertsatz, daß die Mittelwerte annähernd normalverteilt sind und zwar um só besser, je mehr Glieder zur Mittelwertbildung benutzt werden.

4. Die Implementierung der unter Zugrundelegung der Fehlerverteilungen neu berechneten Kontrollgrenzen verhindert, daß zu häufig falsche Alarmmeldungen ausgelöst werden, weil bei der Normalverteilung die Kontrollgrenzen wesentlich enger gefaßt sind.

Für die praktische Anwendung unserer Ergebnisse bieten sich zwei Möglichkeiten an:

\section{Literatur}

1. Domesle, A., Harm, K., Kolster, W. \& Rehpenning, W. (1976), in Organisation des Laboratoires et Interprétation des Résultats - Biologie Prospective, S. 177-183, L'Expansion Scientifique Française, Paris.

2. Domesle, A., Harm, K., Kolster, W. \& Rehpenning, W. (1976), 2nd European Congress on Clinical Chemistry, Prag.

3. Rehpenning, W., Domesle, A., Harm, K. \& Kolster, W. (1977), diese Z. 15, 182.

4. Kolster, W. \& Lensch, S. (1974), Eppendorfer Labor-, Informations- und Aufnahmesystem ELIAS, AEG-Telefunken Druckschrift A511.13.04/04.74, Konstanz.

5. Harm, K. (1974), Med. Progi. Technol. 3, 45-55.

6. Lubran, M. \& Martens, V. (1968), in Technicon Symposia 1967, Automation in Analytical Chemistry, Bd. 1, S. 67-71, Mediad Inc., New York.

7. Büttner, H. (1968), diese Z. 7, 89=95.
1. Für den Fall, daß kein Großrechner zur Verfügung steht, sollten zuerst die Kontrolldaten bei unverändertem Probenzuordnungsmuster langfristig gespeichert werden.

Danach empfehlen wir, die Fehlerverteilungen mit einer Gram-Charlier'schen-Reihe vom Typ A mit möglichst wenigen Summanden darzustellen. Die Approximation gelingt aber nur in einem entsprechend kleinen Bereich (f $\sim 3,0-3,5$ ).

Hierfü sind nur elementare Rechenoperationen erforderlich. Zur Bestimmung der gewünschten Faktoren (Tab. 3, Spalten 3-5) ist die Berechnung der Fläche unter der Dichtefunktion notwendig. Für praktische Zwecke dürfte es in diesem Fall genügen, die Flächenberechnungen planimetrisch durchzuführen oder aber approximativ nach der Simpson-Regel.

2. Soll hingegen die Fehlerverteilung genauer durch unseren letzten Ansatz beschrieben werden, kann auf den Einsatz eines Großrechners nicht verzichtet werden. Die für die Durchführung der Rechnungen erforderlichen FORTRAN-Programme werden auf Anforderung von uns zur Verfügung gestellt.

Für den ersten Ansatz sollten mindestens 300, für den zweiten Ansatz mindestens 1000 Kontrolldaten vorliegen.
8. SMA 12/60 Instruction Manual T-67-109-A (1968), Technicon Corporation, Tarry town.

9. Documenta Geigy, Wissenschaftliche Tabellen (1975), 7. Ausgabe, S. 172, Georg Thieme Verlag, Stuttgart.

10. Cramér, H. (1946), Mathematical Methods of Statistics, Princeton Univ. Press.

11. Kendall, M. G. \& Buckland, W. R. (1975), A Dictionary of Statistical Terms, 3. Aufl., Longman Group Ltd., London.

12. Walsh, G. R. (1975), Methods of Optimization, S. 108-110, John Wiley \& Sons, London-New York-Sidney-Toronto.

13. Marquardt, D. W. (1963), J. Soc. Ind. Appl. Math. 11, $431-441$.

14. Nelder, J. A. \& Mead, R. (1965), Comput. J. 7, 308-313.

15. Rehpenning, W. (1976), Statistische Untersuchung über die Realität einiger Sternringe, Dissertation, Münster.
Priv.-Doz. Dr. med. K. Harm Universitäts-Krankenhaus Eppendorf Zentrallabor der Med. Kliniken Martinistraße 52

D-2000 Hamburg 20 
